\title{
Meta-analysis of the potential role of extracorporeal shockwave therapy in osteonecrosis of the femoral head
}

\author{
Yangquan Hao ${ }^{*^{*}+}$, Hao Guo ${ }^{1 \dagger}$, Zhaochen Xu', Handeng Qi ${ }^{2}$, Yugui Wang ${ }^{2}$, Chao Lu', Jie Liu² and Puwei Yuan ${ }^{2 *}$
}

\begin{abstract}
Background: We aimed to evaluate the role of extracorporeal shockwave therapy (ESWT) in improving osteonecrosis of the femoral head (ONFH).

Methods: We searched studies focusing on the role of ESWT in ONFH using PubMed, Embase, the Cochrane Library, WanFang, VIP, and CNKI databases updated up to July 28, 2017, without language restriction. Standardized mean difference (SMD) values and 95\% confidence intervals (95\% Cls) were pooled to compare the pain score and Harris hip score for ESWT treatment and other treatment strategies.

Results: Four articles, including $230 \mathrm{ONFH}$ patients, were eligible for the meta-analysis. No significant differences were found in the pain score $(S M D=-1.0104 ; 95 \% \mathrm{Cl}-2.3279-0.3071)$ and Harris hip score (SMD $=0.3717 ; 95 \% \mathrm{Cl}$ $-0.3125-1.0559)$ between the two groups before treatment. After treatment, significant differences were found between the experimental and control groups in the pain score (SMD $=-2.1148 ; 95 \% \mathrm{Cl}-3.2332-0.9965)$ and Harris hip score $(S M D=2.1377 ; 95 \% \mathrm{Cl} 1.2875-2.9880)$. There were no significant differences in pain score before and after treatment between the two groups $(S M D=-0.7353 ; 95 \% \mathrm{Cl}-2.1272-0.6566)$, but significant differences were found in the Harris hip score (SMD $=1.2969 ; 95 \%$ Cl 0.7171-1.8767).

Conclusion: For patients at an early stage, ESWT may be safe and effective for relief of pain and improvement of motor function.
\end{abstract}

Keywords: Osteonecrosis of the femoral head, Extracorporeal shockwave therapy, Pain score, Harris hip score, Meta-analysis

\section{Background}

Osteonecrosis of the femoral head (ONFH) is a pathological process that follows ischemic insult [1]. High morbidity occurs in both young and old worldwide [2]. In China, 8.12 million patients have been diagnosed with ONFH as of 2017 [3, 4], and the average annual number of new cases in Korea was 14,103 [5]. The occurrence of osteonecrosis is associated with various risk factors including trauma, hip surgery, corticosteroid use, alcoholism, and coagulopathy [6]. The treatment of ONFH

\footnotetext{
* Correspondence: yangquanhaop17@21cn.com; spine_surgeon@163.com ${ }^{\dagger}$ Yangquan Hao and Hao Guo contributed equally to this work.

${ }^{1}$ Department of Osteonecrosis and Joint Reconstruction, Honghui Hospital Xi'an Jiao Tong University Health Science Center, No. 555 Youyi East Road, Xi'an, Shaanxi 710054, People's Republic of China

${ }^{2}$ Shaanxi University of Chinese Medicine, Shiji Ave, New Economic Zone, Xi'an-Xianyang, Shaanxi 712046, People's Republic of China
}

remains a challenge, and a standardized and improved treatment strategy for ONFH is urgently needed.

"Joint-preserving" treatments, including both surgical (such as core decompression, trochanteric rotational osteotomy, and vascularized bone grafts) and conservative approaches [extracorporeal shock wave therapy (ESWT) and pulsed electromagnetic field] have been developed to prevent progression of ONFH [7, 8]. ESWT is used in physical therapy, orthopedics, urology, and cardiology, and a previous study demonstrated that the technology can successfully treat ONFH [9]. However, the efficacy of ESWT compared with other treatments remains unclear $[10,11]$. For example, no significant difference in efficacy was found between ESWT and core decompression in a study by Wang et al. [10]. A study by Chen and colleagues demonstrated better outcomes with ESWT than with physical 
therapy [12]. Although the effect of ESWT in the treatment of ONFH has been investigated by previous researchers, there is no consistent conclusion about its efficacy when compared with other treatments.

This meta-analysis was performed to evaluate the role of ESWT in improving ONFH. Standardized mean difference (SMD) values and $95 \%$ confidence intervals (95\% CIs) were pooled to compare the pain score and Harris hip score for ESWT treatment and other treatment strategies.

\section{Methods}

\section{Study selection}

Studies were selected using PubMed (http:// www.ncbi.nlm.nih.gov/pubmed), Embase (http://www.embase.com), the Cochrane Library (http://www.cochranelibrary.com), WanFang, VIP, and CNKI databases updated to July 28, 2017, without language restriction. A combination of Medical Subject Headings (MeSH) terms and free-text keywords were used for study selection: ("ESWT" OR "Extracorporeal shock wave") AND ("osteonecrosis" OR "Osteonecrosis" OR "femoral head necrosis" OR "ONFH" OR "Osteonecrosis of the Femoral Head" OR "avascular necrosis of femoral head" OR "necrosis of the femoral head" OR "avascular necrosis of bone" OR "Kienbock disease" OR "Aseptic necrosis of bone").

\section{Selection criteria}

Literature focusing on the efficacy of ESWT in patients with femoral head necrosis were included in the meta-analysis. Studies were included in the meta-analysis if they met the following criteria: (1) published Chinese or English language literature focusing on the efficacy of ESWT in patients with ONFH, in which the experimental group was treated with ESWT and the control group received a different treatment strategy; (2) reported outcomes included the pain score and Harris hip score at baseline and corresponding scores after a period of treatment; and (3) research designed as an interventional study.

Exclusion criteria were as follows: (1) incomplete data or data that could not be used for statistical analysis and (2) reviews, letters, and comments. In addition, if studies duplicated published literature or data for the same population, only the latest research with the most comprehensive information was included.

\section{Data extraction and quality evaluation}

The authors independently extracted the following data from the included literature: the first author's name, year of publication, study period, stage of ONFH (according to Association Research Circulation Osseous), type of study, follow-up duration, baseline characteristics of enrolled patients (e.g., sex ratio, age composition), baseline pain and Harris hip scores, and corresponding scores after treatment, sample sizes, and general demographic data.
The quality of randomized controlled trials was evaluated using Cochrane Collaboration recommendations [13]. Disagreements were resolved by discussion or by consultation with a third reviewer.

\section{Statistical analysis}

Meta-analysis was performed using R 3.12 software ( R Foundation for Statistical Computing, "meta" package, Beijing, China). The SMD values and 95\% CIs were pooled to compare the pain score and Harris hip score for ESWT treatment and other treatment strategies. For $P<0.05$ or $I^{2}>50 \%$, the random effects model was used to calculate the combined effect value. Otherwise, the fixed effects model was chosen to combine data [14]. Publication bias was assessed using Egger's method. Finally, sensitivity analysis was performed by omitting one study at a time to determine the effect on the overall SMD value.

\section{Results}

\section{The general characteristics of included studies}

The flow chart used for study selection is shown in Fig. 1. Of 482 articles initially reviewed, 48 were from PubMed, 91 from Embase, 4 from the Cochrane Library, 61 from WanFang, 46 from VIP, and 232 from CNKI. After excluding duplicated literature, 295 articles were left. Then, the title and abstract were reviewed, and 221 articles obviously inconsistent with the inclusion criteria excluded. Subsequently, a total of 74 articles were fully reviewed, and 52 articles were excluded including 9 letters, 14 reviews, 10 case series/reports, and 19 animal studies. Moreover, another 18 articles including 9 articles without relevant data, 6 descriptive studies, and 3 reduplicative studies were excluded. Finally, 4 articles were included in the meta-analysis $[10,12,14,15]$.

A total of 230 patients (185 men and 45 women, a significant difference) with ONFH were enrolled in this study, including 120 in the experimental group and 110 in the control group. The general characteristics of the selected literature are shown in Table 1. The publication year ranged from 2008 to 2015. Patients with ONFH were mainly in stages I-III. Only one randomized controlled study was included. No significant difference in sex or age distribution was found in individual studies. Follow-up time in three studies was more than 1 year. Figure 2 shows that the quality of the included literature was relatively poor.

\section{Meta-analysis of pain score and Harris hip score}

The pain and Harris hip scores before and after treatment in the experimental and control groups were analyzed. The main results are shown in Table 2 and Fig. 3. No significant differences were found between the two groups in the baseline pain score $(\mathrm{SMD}=-1.0104 ; 95 \%$ CI - 2.3279-0.3071) and baseline Harris hip score 


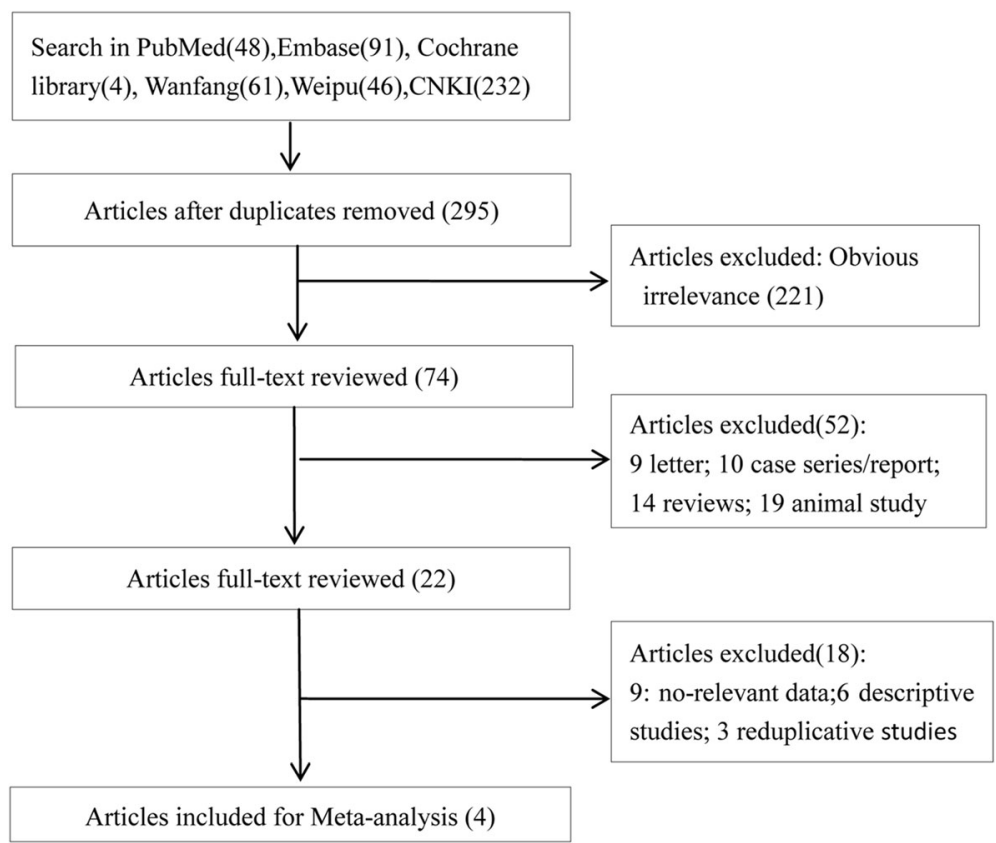

Fig. 1 Flow chart of study selection

$(\mathrm{SMD}=0.3717 ; 95 \% \mathrm{CI}-0.3125-1.0559)$. After a period of treatment, significant differences were found between the experimental and control groups in the pain score $(\mathrm{SMD}=-2.1148 ; 95 \% \mathrm{CI}-3.2332-0.9965)$ and Harris hip score $(\mathrm{SMD}=2.1377 ; 95 \%$ CI $1.2875-2.9880)$. No significant differences in pain score were found before and after treatment (SMD $=-0.7353 ; 95 \% \mathrm{CI}-2.1272$ 0.6566), but significant differences were found in the Harris hip score (SMD $=1.2969 ; 95 \%$ CI 0.7171-1.8767).

\section{Publication bias}

Significant bias was found among individual studies in the comparison of Harris hip scores $(t=3.5824, P=0.0231)$, but no publication bias was found in the change before and after treatment $(t=0.9755, P=0.3846)$ in the baseline pain score and Harris hip score $(t=1.9243, P=0.1267)$.

\section{Sensitivity analysis}

Sensitivity analysis of pain scores demonstrated that the results were unstable, but sensitivity analysis of Harris hip scores demonstrated that the results were stable.

\section{Discussion}

In the present study, we evaluated the role of ESWT in improving ONFH. A total of 230 patients with ONFH were included in the study. No significant difference was found between the two groups in the pain score and Harris hip score before treatment. After treatment, significant differences were found between the experimental and control groups in the pain score and Harris hip score. No significant differences in pain score were found before and after treatment, but significant differences were found in the Harris hip score.

Table 1 The baseline characteristics of included studies

\begin{tabular}{|c|c|c|c|c|c|c|c|c|c|}
\hline Study & Year & Study year & ONFH stage & Study style & Group & Number/hips & Gender (M/F) & Age (year) & Duration (month) \\
\hline \multirow[t]{2}{*}{ Wang CJ } & 2012 & $2001-2001$ & Stages I, II, early III & Non-RCT & ESWT & $23 / 29$ & $20 / 3$ & $39.8 \pm 12.1$ & $25.2 \pm 3.7$ \\
\hline & & & & & Surgical group & $25 / 28$ & $22 / 3$ & $39.9 \pm 9.3$ & $25.8 \pm 4.6$ \\
\hline \multirow[t]{2}{*}{ Chen JM } & 2009 & 1999.7-2006.1 & Stages I-III & Non-RCT & ESWT & $17 / 17$ & $14 / 3$ & $42.9 \pm 9.3$ & $11.3 \pm 3.4$ \\
\hline & & & Stages I-IV & & THA & $17 / 17$ & $14 / 3$ & $42.9 \pm 9.3$ & $14.7 \pm 0.93$ \\
\hline \multirow[t]{2}{*}{ Zhang HJ } & 2015 & 2009.1-2012.12 & Stages I-II & $\mathrm{RCT}$ & BMSC+ESWT & $20 / 29$ & $15 / 5$ & $36.1 \pm 6.2$ & 24 \\
\hline & & & & & BMSC & $20 / 27$ & $14 / 6$ & $35.5 \pm 5.7$ & 24 \\
\hline \multirow[t]{2}{*}{ Zhai $L$} & 2008 & 1998.1-2007.6 & Stages I-III & Non-RCT & $C D+E S W T$ & $50 / 50$ & $41 / 9$ & $20.9(18-25)$ & $6.4(3-18)$ \\
\hline & & & & & $C D$ & $58 / 58$ & $45 / 13$ & $20.5(18-25)$ & $7.3(3-20)$ \\
\hline
\end{tabular}

ESWT extracorporeal shock wave treatment, THA total hip arthroplasty, CD core decompression, $M / F$ males/females, $R C T$ randomized controlled trail, ONFH osteonecrosis of the femoral head, THA total hip, BMSC bone marrow stem cells 


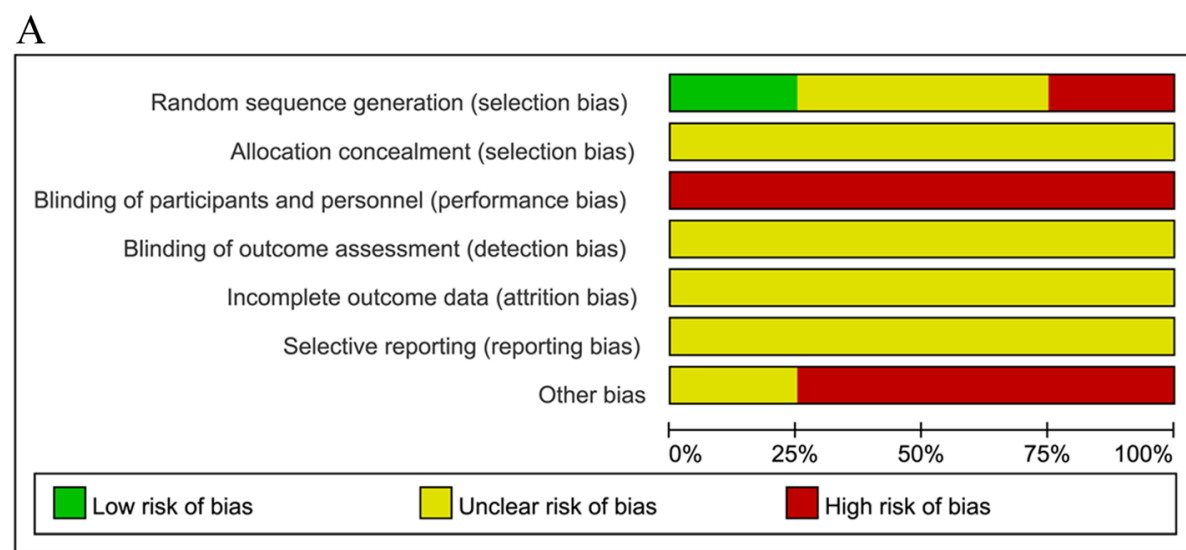

B

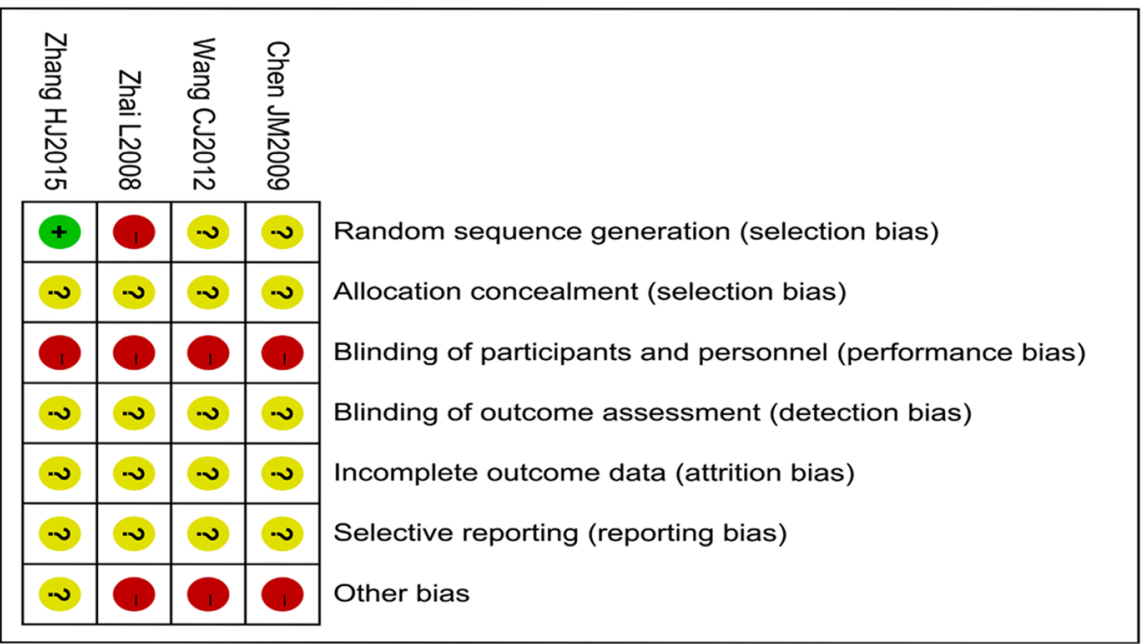

Fig. 2 Quality assessment of the meta-analysis. a Risk of bias. $\mathbf{b}$ risk of bias summary

Physical therapy can improve bone oxygenation, reduce edema, reduce bone pressure, improve bone circulation, prevent ischemia, restore blood supply in hypoxic tissue, and promote necrotic bone repair [16]. Wang et al. demonstrated greater improvement with ESWT compared with core decompression and nonvascularized fibular grafting in patients with early-stage ONFH [17].
A previous study demonstrated that ESWT could increase the expression of angiogenic factors, reduce vessel wall stenosis, and improve limb perfusion [18]. The effect of ESWT might be related to stress-induced piezoelectricity, cavitation and osteogenesis, and metabolic activation. These effects promote healing of femoral head necrosis by inducing improved blood circulation,

Table 2 Meta-analysis results for pain score and Harris hip score

\begin{tabular}{|c|c|c|c|c|c|c|c|c|c|c|c|c|c|}
\hline \multirow[t]{2}{*}{ Variable } & \multirow[t]{2}{*}{ Group } & \multicolumn{3}{|c|}{ Sample size } & \multicolumn{3}{|l|}{ Test of association } & \multirow[t]{2}{*}{ Model } & \multicolumn{3}{|c|}{ Test of heterogeneity ${ }^{a, b}$} & \multicolumn{2}{|c|}{ Egger's test $^{\mathrm{C}}$} \\
\hline & & $\bar{K}$ & ESWT & $\overline{\text { Control }}$ & SMD (95\% Cl) & Z & $P$ & & $\bar{Q}$ & $P$ & $P^{2}(\%)$ & $t$ & $P$ \\
\hline \multirow[t]{3}{*}{ Pain score } & Base & 2 & 46 & 45 & $-1.0104[-2.3279 ; 0.3071]$ & 1.5032 & 0.1328 & Random & 6.61 & 0.0058 & 86.9 & - & - \\
\hline & Post & 2 & 46 & 45 & $-2.1148[-3.2332 ;-0.9965]$ & 3.7063 & 0.0002 & Random & 4.42 & 0.0035 & 77.4 & - & - \\
\hline & Change & 2 & 46 & 46 & $-0.7353[-2.1272 ; 0.6566]$ & 1.0354 & 0.3005 & Random & 9.81 & 0.0017 & 89.8 & - & - \\
\hline \multirow[t]{3}{*}{ Harris hip score } & Base & 4 & 125 & 130 & $0.3717[-0.3125 ; 1.0559]$ & 1.0647 & 0.287 & Random & 34.06 & $<0.001$ & 85.3 & 1.9243 & 0.12 \\
\hline & Post & 4 & 125 & 130 & $2.1377[1.2875 ; 2.9880]$ & 4.9281 & $<0.001$ & Random & 35.77 & $<0.001$ & 86.0 & 3.5824 & 0.023 \\
\hline & Change & 4 & 125 & 125 & $1.2969[0.7171 ; 1.8767]$ & 4.3839 & $<0.001$ & Random & 20.72 & 0.001 & 75.9 & 0.9755 & 0.38 \\
\hline
\end{tabular}

$O R$ odds ratio, $C l$ confidence interval, $K$ number of studies combined

${ }^{a}$ Random-effects model was used when the $P$ for heterogeneity test $<0.05$; otherwise, the fixed-effect model was used

${ }^{\mathrm{b}} P<0.05$ is considered statistically significant for $\mathrm{Q}$ statistics

${ }^{\mathrm{C}}$ Egger's test to evaluate publication bias, $P<0.05$ is considered statistically significant 


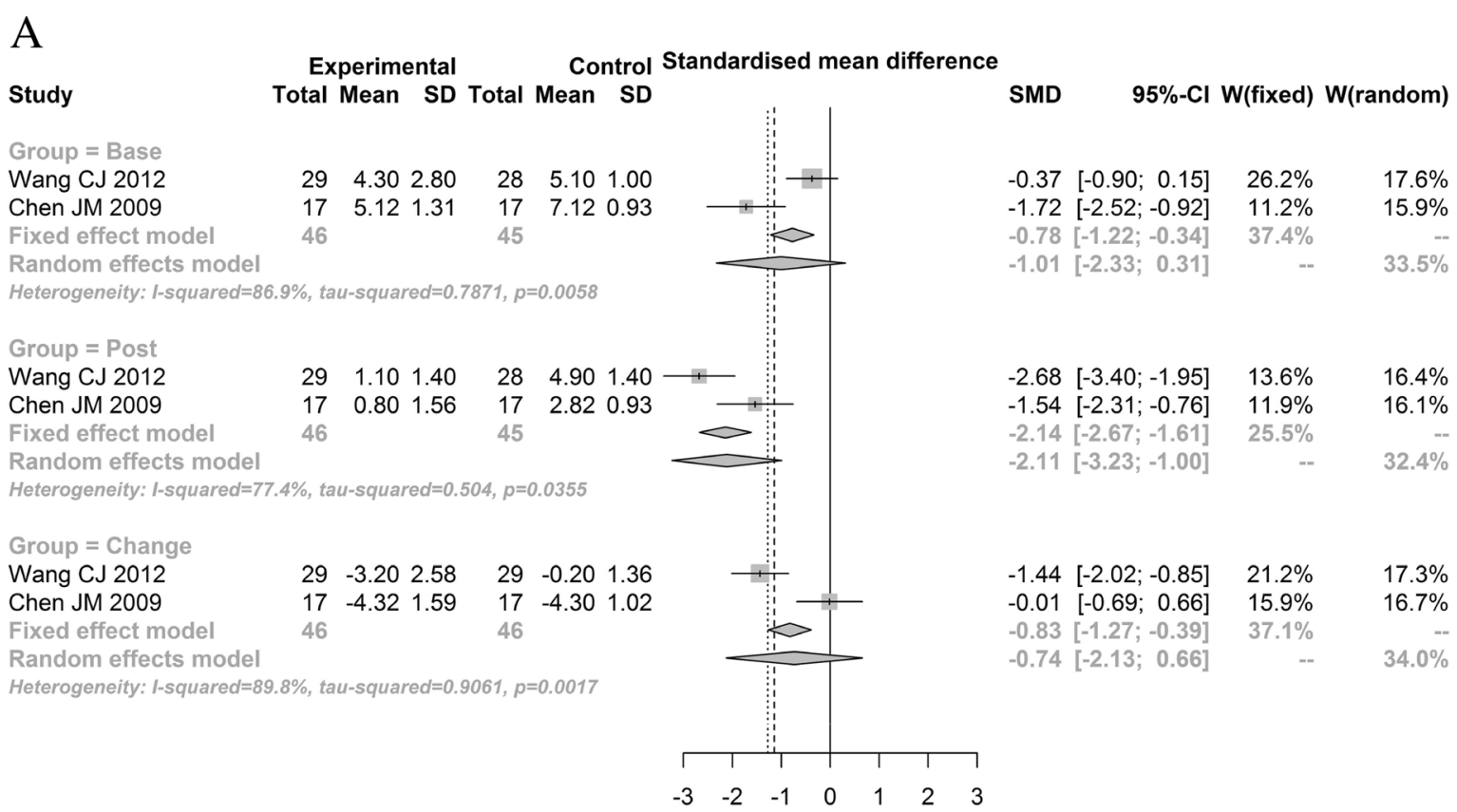

B

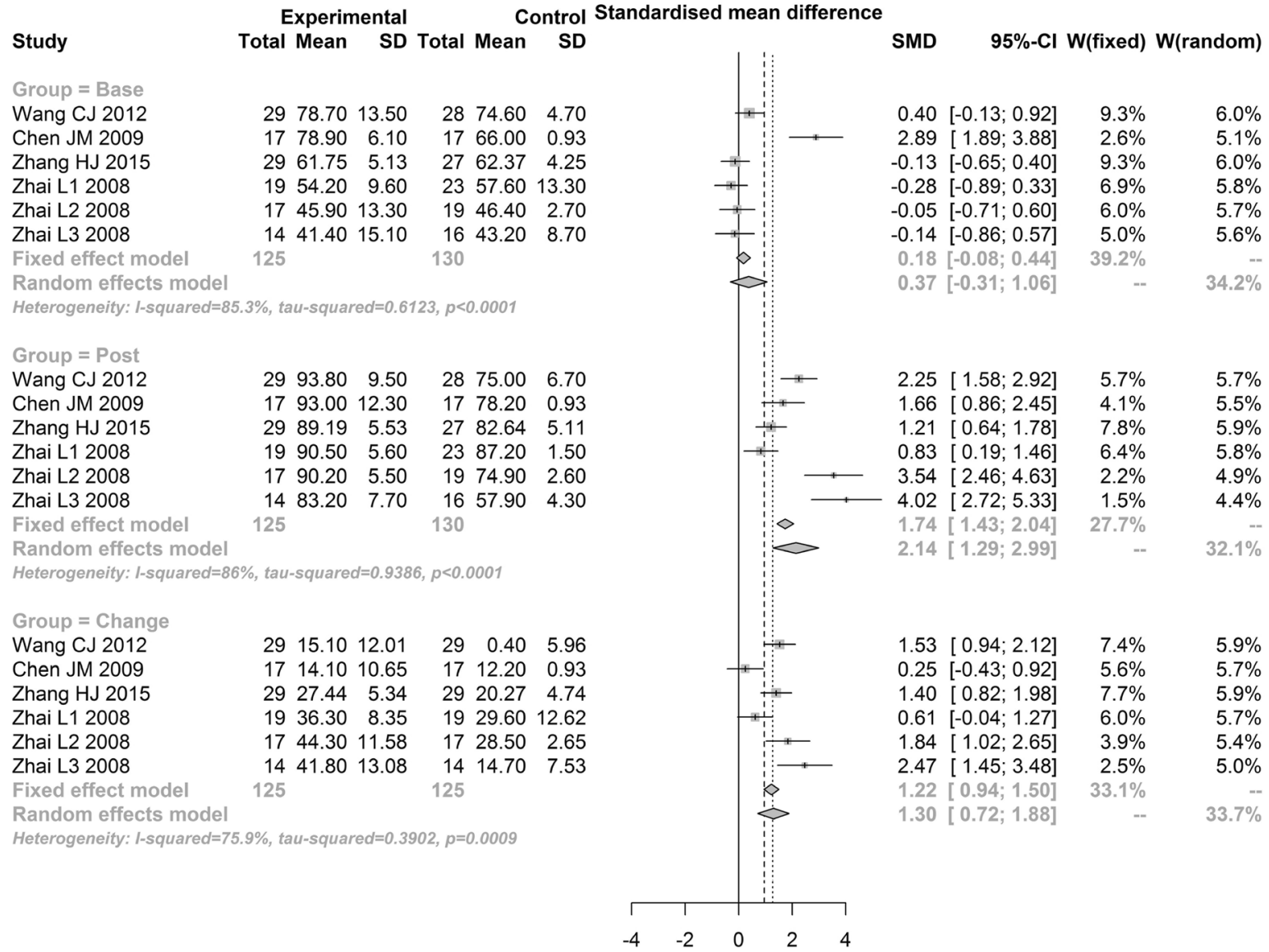

Fig. 3 Meta-analysis of pain score and Harris hip score. a Pain score. $\mathbf{b}$ Harris hip score 
mitigating a hypercoagulable state, and enhancing osteoblast and blood vessel activity $[18,19]$. Similar to previous reports, the present study suggested that ESWT might be a safe and effective method to improve motor function and relieve pain, especially at an early stage of ONFH. Significant heterogeneity was observed in the study. Heterogeneity might be introduced by different combined treatment strategies. For example, patients in experimental groups underwent ESWT in two studies [12], while patients in experimental groups in two other studies underwent combined treatment $[15,20]$. Although the age difference between groups in individual studies was not significant, the age in the four studies ranged from 20.9 to 40.9 years. Bone density, structure, and strength are correlated with age $[21,22]$. Thus, efficacy should be confirmed with further studies after adjusting for background factors that can affect ESWT treatment.

Some limitations should be noted. First, the small sample size introduced more obvious heterogeneity between individual studies [23]. Additionally, the included populations were small and the baseline characteristics of included studies were not complete. Thus, subgroup analysis based on age and sex distribution could not be performed. Second, the quality of the included studies was poor, limiting the strength of the conclusion. Third, publication bias for the Harris hip score after treatment might affect the results. Fourth, only two studies reported pain scores, and further research with larger sample sizes is needed to validate the conclusions.

\section{Conclusion}

For patients at an early stage, ESWT may be a safe and effective way to relieve pain and improve motor function. Nevertheless, due to the low quality of the included publications, the conclusion should be confirmed with further research using a larger sample size. The long-term follow-up studies are favorable to the use of ESWT in $\mathrm{ONFH}$ in future.

\section{Abbreviations \\ Cl: Confidence interval; ESWT: Extracorporeal shockwave therapy; ONFH: Osteonecrosis of the femoral head; SMD: Standardized mean difference}

\section{Acknowledgements}

This work was supported by the key scientific and technological innovation team of Shaanxi province (grant number: 2013KCT-26).

\section{Funding}

This work was supported by key scientific and technological innovation team of Shaanxi province (grant number: 2013KCT-26).

\section{Availability of data and materials}

All data generated or analyzed during this study are included in this published article [and supplementary information files].

\section{Authors' contributions}

YH and PY conceived and designed the research. $J$ acquired the data. YW analyzed and interpreted the data. CL and HG performed the statistical analyses. PY obtained the funding. ZX and HQ drafted the manuscript. YH and PY revised the manuscript for important intellectual content. All authors read and approved the final manuscript.

Ethics approval and consent to participate

Not applicable.

Consent for publication

Not applicable.

\section{Competing interests}

The authors declare that they have no competing interests.

\section{Publisher's Note}

Springer Nature remains neutral with regard to jurisdictional claims in published maps and institutional affiliations.

Received: 4 January 2018 Accepted: 11 June 2018

Published online: 03 July 2018

\section{References}

1. Nakai T, Masuhara K, Nakase T, Sugano N, Ohzono K, Ochi T. Pathology of femoral head collapse following transtrochanteric rotational osteotomy for osteonecrosis. Arch Orthop Trauma Surg. 2000;120(9):489-92.

2. Leclerc C, Néant I, Moreau M. The calcium: an early signal that initiates the formation of the nervous system during embryogenesis. Jorgchem. 2012; 36(18):2720-1.

3. Pascart T, Falgayrac G, Migaud H, Quinchon JF, Norberciak L, Budzik JF, et al. Region specific Raman spectroscopy analysis of the femoral head reveals that trabecular bone is unlikely to contribute to non-traumatic osteonecrosis. Sci Rep. 2017;7(1):017-00162.

4. Zhao DW, Yu M, Hu K, Wang W, Yang L, Wang BJ, et al. Prevalence of nontraumatic osteonecrosis of the femoral head and its associated risk factors in the Chinese population: results from a nationally representative survey. Chin Med J. 2015;128(21):2843-50.

5. Kang JS, Park S, Song JH, Jung YY, Cho MR, Rhyu KH. Prevalence of osteonecrosis of the femoral head: a nationwide epidemiologic analysis in Korea. J Arthroplasty. 2009;24(8):1178-83.

6. Qiang H, Liu H, Ling M, Wang K, Zhang C. Early steroid-induced osteonecrosis of rabbit femoral head and Panax notoginseng saponins: mechanism and protective effects. Evid Based Complement Alternat Med. 2015;2015:719370. https://doi.org/10.1155/2015/719370.

7. Sadile F, Bernasconi A, Russo S, Maffulli N. Core decompression versus other joint preserving treatments for osteonecrosis of the femoral head: a metaanalysis. Br Med Bull. 2016:118(1):33-49.

8. Kuroda Y, Matsuda S, Akiyama H. Joint-preserving regenerative therapy for patients with early-stage osteonecrosis of the femoral head. Inflamm Regen. 2016;36(4):016-0002.

9. Lin PC, Wang CJ, Yang KD, Wang FS, Ko JY, Huang CC. Extracorporeal shockwave treatment of osteonecrosis of the femoral head in systemic lupus erythematosis. J Arthroplast. 2006;21(6):911-5.

10. Wang CJ, Huang CC, Wang JW, Wong T, Yang YJ. Long-term results of extracorporeal shockwave therapy and core decompression in osteonecrosis of the femoral head with eight- to nine-year follow-up. Biom J. 2012;35(6):481-5.

11. Zhang Q, Liu L, Sun W, Gao F, Cheng L, Li Z. Extracorporeal shockwave therapy in osteonecrosis of femoral head: a systematic review of now available clinical evidences. Medicine. 2017;96(4):e5897.

12. Chen JMHS, Wong T, Chou WY, Wang CJ, Wang FS. Functional outcomes of bilateral hip necrosis: total hip arthroplasty versus extracorporeal shockwave. Arch Orthop Trauma Surg. 2009;129:837-41.

13. Higgins JP, Green S. Cochrane handbook for systematic reviews of interventions version 5.1.0: The Cochrane Collaboration; 2011. Available from http://handbook.cochrane.org.

14. Feng R-N, Zhao C, Sun C-H, Li Y. Meta-analysis of TNF 308 G/A polymorphism and type 2 diabetes mellitus. PLoS One. 2011;6(4):e18480

15. Lei ZGX, Chuan J, Bing L, Zhe Z, Xuming W. Therapeutic effects of extracorporeal shock waves combined with drilling decompression for the 
treatment of femoral head ischemic necrosis due to training in servicemen. Med J Chin PLA. 2008;33:348-50.

16. Neumayr LD, Aguilar C, Earles AN, Jergesen HE, Haberkern CM, Kammen BF, et al. Physical therapy alone compared with core decompression and physical therapy for femoral head osteonecrosis in sickle cell disease. Results of a multicenter study at a mean of three years after treatment. J Bone Joint Surg Am. 2006;88(12):2573-82.

17. Wang CJ, Wang FS, Huang CC, Yang KD, Weng LH, Huang HY. Treatment for osteonecrosis of the femoral head: comparison of extracorporeal shock waves with core decompression and bone-grafting. J Bone Joint Surg Am. 2005;87(11):2380-7.

18. Raza A, Harwood A, Totty J, Smith G, Chetter I. Extracorporeal shockwave therapy for peripheral arterial disease: a review of the potential mechanisms of action. Ann Vasc Surg. 2017;45:294-8.

19. Korakakis V, Whiteley R, Tzavara A, Malliaropoulos N. The effectiveness of extracorporeal shockwave therapy in common lower limb conditions: a systematic review including quantification of patient-rated pain reduction. Br J Sports Med. 2018;52(6):387-407.

20. Hongjun ZSW, Kejie F, Shaohui W, Yanzhao Z. Extracorporeal shock wave therapy combined with autologous bone marrow stem cells transplantation for treating early-stage osteonecrosis of the femoral head. Chin J Phys Med Rehabil. 2015;37:287-90.

21. Alvarenga JC, Fuller H, Pasoto SG, Pereira RM. Age-related reference curves of volumetric bone density, structure, and biomechanical parameters adjusted for weight and height in a population of healthy women: an HRpQCT study. Osteoporos Int. 2017;28(4):1335-46.

22. Zura R, Braid-Forbes MJ, Jeray K, Mehta S, Einhorn TA, Watson JT, et al. Bone fracture nonunion rate decreases with increasing age: a prospective inception cohort study. Bone. 2017;95:26-32.

23. IntHout J, loannidis JP, Borm GF, Goeman JJ. Small studies are more heterogeneous than large ones: a meta-meta-analysis. J Clin Epidemiol. 2015;68(8):860-9.

\section{Ready to submit your research? Choose BMC and benefit from:}

- fast, convenient online submission

- thorough peer review by experienced researchers in your field

- rapid publication on acceptance

- support for research data, including large and complex data types

- gold Open Access which fosters wider collaboration and increased citations

- maximum visibility for your research: over $100 \mathrm{M}$ website views per year 